\title{
DENSITY SENSITIVE LINES IN THE EUV REGION
}

J.E. Vernazza

Centre for Astrophysics, Cambridge, Mass., USA

H. E. Mason

Dept. of Applied Mathematics and Theoretical Physics

Silver Street, Cambridge, England

A search for density sensitive line ratios was made in the $1400 \AA$ to $300 \AA$ region of the solar spectra. The emergent intensity of resonance and subordinate lines from ions of the beryllium and boron isoelectronic sequence were calculated for typical solar plasmas. For individual ions, line ratios which are sensitive to. electron densities changes betweer, $10^{7}$ to $10^{13} \mathrm{~cm}^{-3}$ were selected. These line ratios were then compared to observations made by the Harvard College Observatory spectrometer aboard SKYLAB. From all the potential candidates only few are adequately observed to estimate electron densities. In particular the $\mathrm{Mg}$ VII lines at 430 and $436 \mathrm{R}$ are good indicators of the coronal densities. Comparisons of the densities of coronal holes, flaxes, and active regions are made. 